\title{
Architectural Ghosts: Storytelling and the Urban Imaginary
}

\author{
ZOE LYNNE COPE \\ University of Nebraska-Lincoln
}

\begin{abstract}
'Architectural Ghosts' proposes architecture as a series of eleven speculative cities that function as characters engaged in a theatrical masque set in contemporary Rome, Italy. The project challenges traditional methods of architectural preservation and memory by presenting a new way to imagine history at the intersection of narrative and architecture. 'Architectural Ghosts' seeks to qualify experiences of architecture, performance and the social imaginary as catalyzed by the urban environment.
\end{abstract}

A re-imagining of the novel Invisible Cities by Italo Calvino, this project utilizes narrative storytelling as architectural medium, method and site. The framework of the original novel was analyzed, collapsed and re-written in a contemporary context. The project offers a critique of the use of the novel in architectural education and challenges the marginalized role of women presented in both the original text and throughout much of architectural history.

Calvino's cities were metaphors for women conquered by two powerful male explorers. In this project, each city was designed and illustrated relative to site, theme and the work of a prominent female storyteller, philosopher, or architect as a way to presence their ghosts. Much of the research was oriented around unearthing the accomplishments of women engaged in a partnership wherein a male counterpart received credit for much of the work. The plot is structured by an overarching conversation between a female narrator and a male character. Desire for what her voice brings into being creates a tension that drives the entirety of the plot, representation and sequencing of architectural experiences. Eventually, it is revealed that she herself is also a city; she too is a ghost catalyzed by architectural and phenomenological experiences that imply presence.

This project explores 'architectural ghosts' as allegory for the presence of the absence of presence that reveals architecture as performance. The universal gap between lived experience and representation is explored as primary function of the paradox of human desire. Literary narrative is utilized as structure for the gap of philosophical lack and as active agent for the discrete, conditional alignments that generate and inform productive encounters with the architectural imaginary. The masque, as performance between complex and layered characters, reveals the individual and collective nature of architecture, cities and the stories we tell about both. 

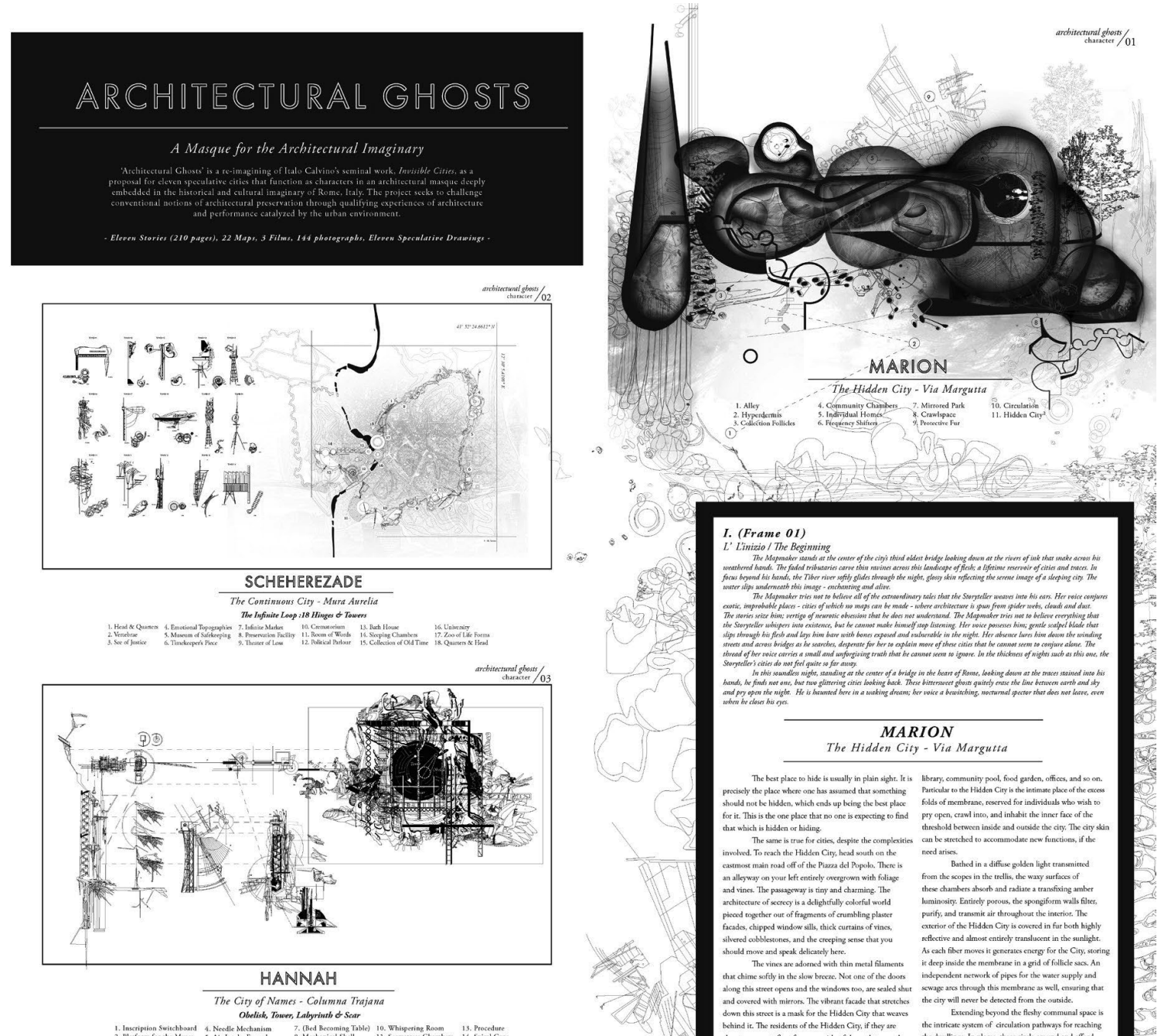

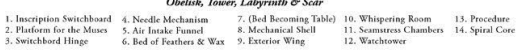

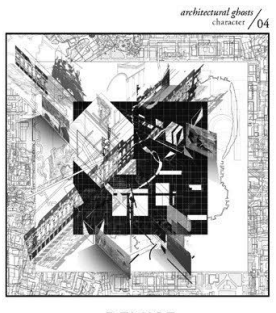

DENISE

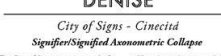

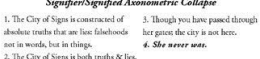
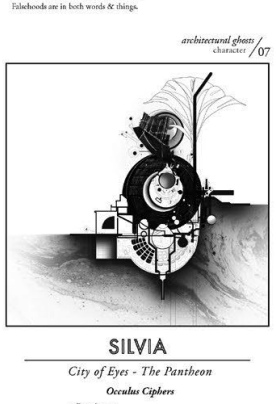

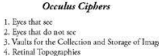

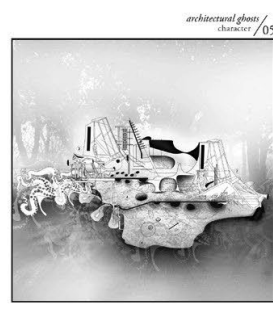

SIMONÉ

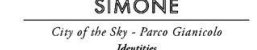

E
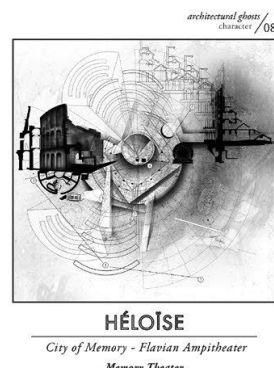

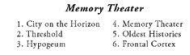
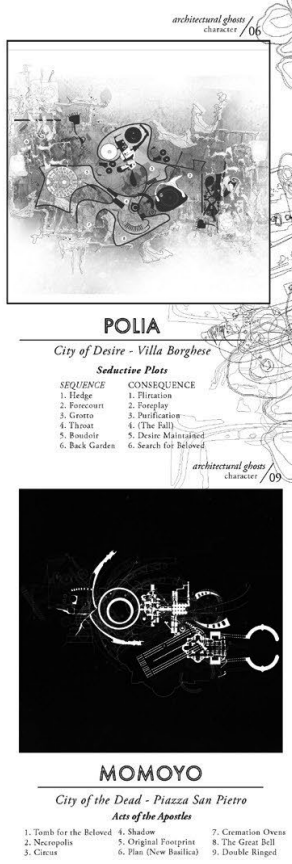
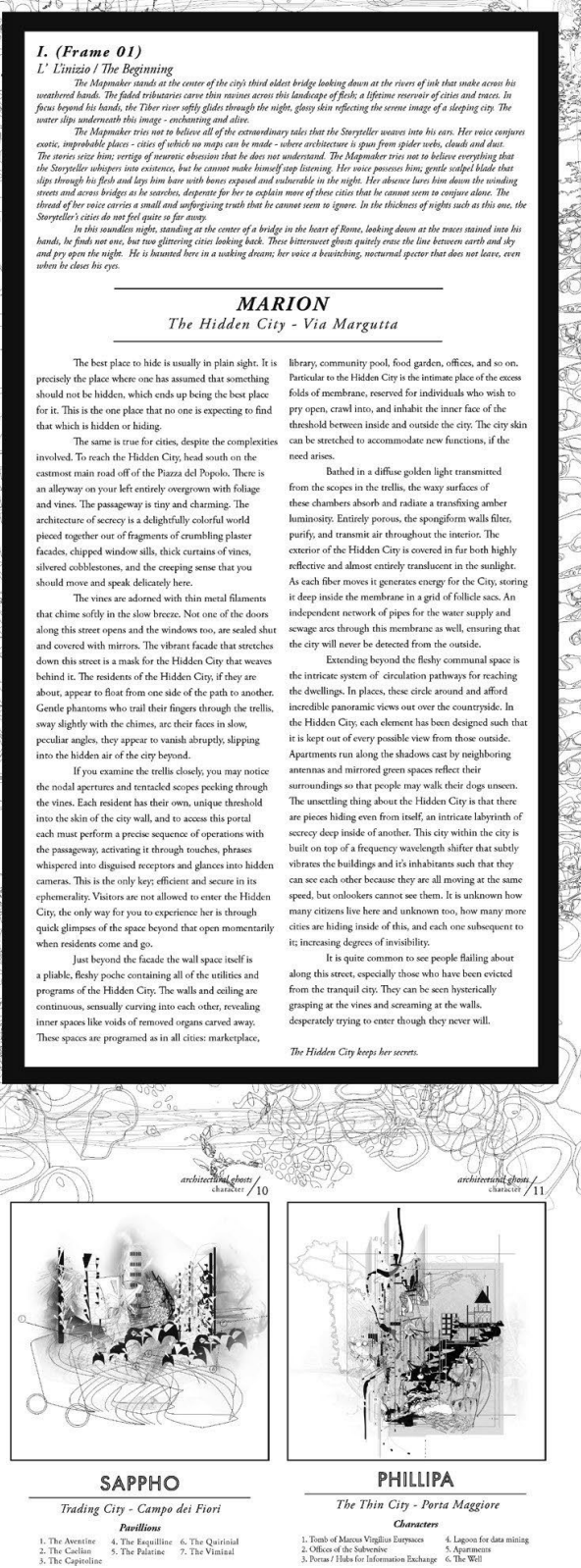

$\frac{\text { PHILLIPA }}{\text { The Thin City - Porta Maggiore }}$

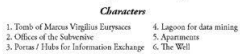

